\title{
Association between serum magnesium and blood lipids: influence of type 2 diabetes and central obesity
}

\author{
Lianlong $\mathrm{Yu}^{1 *}$, Junli Zhang ${ }^{1} \dagger$, Liansen $\mathrm{Wang}^{1} \dagger$, Suyun $\mathrm{Li}^{1} \dagger$, Qian Zhang ${ }^{2} \dagger$, Peirui Xiao ${ }^{1} \dagger$, Kebo $\mathrm{Wang}^{1} \dagger$, \\ Maoqiang Zhuang ${ }^{1} \dagger$ and Ying Jiang $^{1} \dagger$ \\ ${ }^{1}$ Shandong Center for Disease Control and Prevention, Institution of Food and Nutrition, 16992 Jingshi Road, Jinan, \\ Shandong 250014, People's Republic of China \\ ${ }^{2}$ Office of Radiation Hygiene, Shandong Agency for Health and Family Planning Inspection, 75 Yuhan Road, Jinan, \\ Shandong 250000, People's Republic of China
}

(Submitted 29 October 2017 - Final revision received 10 February 2018 - Accepted 19 February 2018 - First published online 23 May 2018 )

\section{Abstract}

To assess the changes in the relationship between serum Mg and blood lipids of Chinese adults with type 2 diabetes (T2D) or central obesity, a total of 8163 subjects (mean age 59.6 years, $54.9 \%$ men) were analysed. Participants were classified according to blood Mg (below 0.65 mmol/1, 0.65-0.95 mmol/1 and above $0.95 \mathrm{mmol} / \mathrm{l}$ ), T2D (yes/no) and central obesity (yes/no). Blood lipids (TAG, total cholesterol (TC), HDL-cholesterol and LDL-cholesterol) were determined by standardised methods and conditions. A significant increase in blood lipids, with the exception of HDL-cholesterol, across progressive Mg groups in all subjects was noted $(P<0.05)$. TAG, TC, HDL-cholesterol and LDL-cholesterol were significantly higher among subjects with central obesity compared with those without central obesity $(P<0 \cdot 05)$. A significant increase in TAG, TC and LDL-cholesterol across progressive Mg groups was observed in subjects without T2D $(P<0 \cdot 05)$. However, no significant change of HDL-cholesterol and LDL-cholesterol across progressive Mg groups was observed in subjects with T2D $(P>0 \cdot 05)$. TAG, TC, HDL-cholesterol and LDL-cholesterol were significantly higher among subjects with T2D than those without T2D $(P<0 \cdot 05)$. Multivariable models for TAG and LDLcholesterol failed to attain statistical significance in diabetics, by using a generalised linear or parsimonious model. TAG, TC, HDL-cholesterol and LDL-cholesterol were significantly higher among subjects with T2D or central obesity. Blood lipids, with the exception of HDL-cholesterol, were associated with serum Mg, but this association was somehow influenced by T2D in LDL-cholesterol. In addition, multivariable models for both TAG and LDL-cholesterol failed to attain statistical significance among subjects with T2D, different from subjects without T2D.

\section{Key words: Blood lipids: Magnesium: Central obesity: Type 2 diabetes}

Mg plays a key role in a wide range of cellular functions, which are known to affect many aspects of the endocrine system ${ }^{(1-4)}$. Many studies showed that lower serum or urinary $\mathrm{Mg}$ was associated with an increased risk of ischaemic stroke ${ }^{(5)}$, CHD and cardiovascular mortality ${ }^{(6,7)}$. However, serum $\mathrm{Mg}$ concentrations were negatively associated with type 2 diabetes (T2D) and indices of glycaemic control and insulin resistance ${ }^{(8)}$.

Some studies ${ }^{(9,10)}$ have shown that dyslipidaemia is one of the known risk factors for T2D. Traditional lipid measures, including total cholesterol (TC), TAG, HDL-cholesterol and LDL-cholesterol, are well documented in their association with the incidence of $\mathrm{T} 2 \mathrm{D}^{(11,12)}$.

In addition, central obesity had a greater effect on TC and LDL-cholesterol than BMI among women ${ }^{(13)}$. The highest mean of serum lipids and the highest extent of dyslipidaemia existed in children having central obesity along with general obesity ${ }^{(14)}$.
In the study by Cao et al. ${ }^{(15)}$, higher serum $\mathrm{Mg}$ levels were associated with higher TC, TAG, HDL-cholesterol and LDL-cholesterol but lower non-HDL-cholesterol/HDLcholesterol in the total population (all $P<0.05$ ). Similar relationships of urinary $\mathrm{Mg}$ with lipoproteins were also found in this study (all $P<0 \cdot 05$ ). In contrast, in the study by Shahbah et al. ${ }^{(16)}$, serum Mg was found to be positively correlated with HDL $(P<0.001)$ and negatively correlated with TAG, TC and LDL-cholesterol $(P<0.001)$. In addition, the study by Rasheed et $a l .{ }^{(17)}$ found that mean serum $\mathrm{Mg}$ in diabetic patients was significantly lower as compared with healthy subjects.

Some of the conflicting study results confused us, which is attributed to their insufficient adjustment for confounders. Meanwhile, limited studies examined the association between serum Mg and blood lipids in subjects with T2D and central obesity.

Abbreviations: T2D, type 2 diabetes; TC, total cholesterol; WC, waist circumference.

* Corresponding author: L. Yu, fax +860531 82679425, email lianlong00a@163.com

$\dagger$ These authors contributed equally to this work and should be considered co-first authors. 
The aim of our study was to explore the changes in the relationship between blood lipids and serum $\mathrm{Mg}$ of Chinese adults with T2D or central obesity. Using the Metabolic, Lifestyle and Nutrition Assessment in adult survey-China Health and Nutrition Survey (CHNS), we report that an elevated TAG, TC and LDLcholesterol increased across progressive $\mathrm{Mg}$ groups in some subgroups. However, this association is somehow influenced by T2D. Meanwhile, after adjusting for confounders, a new phenomenon about serum lipids is found in lower serum $\mathrm{Mg}$ levels.

\section{Methods \\ Study design}

The CHNS is a large-scale, national cross-sectional survey that was designed to investigate the Chinese health and nutritional status. Currently, data are available in 2009. A stratified multi-stage cluster random process was used to draw samples from nine provinces of China, which included Shandong, Henan, Liaoning, Hunan, Heilongjiang, Jiangsu, Hubei, Guangxi and Guizhou. All participants voluntarily joined this study with informed consent, and the study was approved by an institutional review board of the University of North Carolina at Chapel Hill and China Center for Disease Control and Prevention. There was no Mg Supplementation during this study.

\section{Study population}

Participants aged 18 years or older were included in this study. Information on age, sex, region, activity level and dietary behaviour was collected. There were 8163 participants included in the surveys organised in 2009. This sample is varied, with variation found in a wide range of related biochemical markers, healthy factors, nutritional and demographic measures ${ }^{(18)}$, TAG, TC, HDL-cholesterol and LDL-cholesterol.

The current study is a cross-sectional analysis of 8163 subjects included in the surveys organised in 2009, with complete information for the determination of T2D, central obesity, serum lipids and blood $\mathrm{Mg}$, as described below. Every subject underwent the same examinations during this survey.

\section{Measurements and definitions}

Individuals aged over 7 years went to a neighbourhood clinic to have fasting blood samples collected by trained physicians. Individuals who were unable to attend the clinic had blood samples collected at home. To avoid omitting children in boarding schools and migrant workers, special efforts were made to schedule visits early in the morning or on weekends when these participants were at home. All interviewers spent $7 \mathrm{~d}$ in training provided by the collaborating teams before the survey, and were overseen through the Internet during their visits (at the same time, the monitor data were collected by the University of North Carolina at Chapel Hill, the China Centers for Disease Control and Prevention and the China-Japan Friendship Hospital).

Systolic (SBP) and diastolic (DBP) blood pressure was measured on the right arm, using mercury Sphygmomanometers with appropriate cuff sizes. Blood pressure was measured three times over a 10-min seated rest, and the mean of the three measurements was used in our analyses. The time interval between two measurements was about $30 \mathrm{~s}$.

Testing of blood cell count was to be completed in local laboratories in accordance with the consensus guidelines of CHNS. Testing of serum lipids and blood Mg was to be completed in China-Japan Friendship Hospital, Ministry of Health laboratory, which meets all requirements for accurate measurement and testing.

After an overnight fast, blood was collected by venepuncture and tested immediately for glucose and HbA1c. Plasma and serum samples were then frozen and stored at $-86^{\circ} \mathrm{C}$ for later laboratory analysis. All samples were analysed in a national central laboratory in Beijing (medical laboratory accreditation certificate ISO 15189:2007) with strict quality control. We focus on blood pressure (SBP and DBP) and biomarkers of cardiometablic disease risk related to T2D, dyslipidaemia (TC, HDL-cholesterol and LDL-cholesterol all measured using glycerol-phosphate oxidase method and the PEG-modified enzyme method, respectively, by determiner reagents (Kyowa Medex Co., Ltd) and TAG using glycerol-phosphate oxidase method and the PEG-modified enzyme method, respectively, by determiner regents (Kyowa Medex Co., Ltd)). Mg was measured on the Hitachi 7600 automated Analyzer Randox (Hitachi Inc.) by the Xylidyl Blue Colorimetric method (Randox Laboratories Ltd.

Weight was measured to the nearest $0 \cdot 1 \mathrm{~kg}$ with light-weight clothing on a calibrated beam scale, and height was measured to the nearest $0 \cdot 1 \mathrm{~cm}$ without shoes with a portable stadiometer. BMI was calculated as weight in kilograms divided by the square of height in metres. Waist circumference (WC) was measured at a point midway between the lowest rib and the iliac crest in a horizontal plane using non-elastic tape. Height, weight and WC were measured by trained examiners following a standard protocol from the $\mathrm{WHO}^{(19)}$. Height, weight and WC measurements were made at the same location and the same protocol was followed at each study visit.

Central obesity was defined by WC $>90 \mathrm{~cm}$ for men and $>80 \mathrm{~cm}$ for women ${ }^{(20)}$. Laboratory data were obtained within 2 months of the questionnaire visit.

Subjects were classified according to T2D (yes/no) and central obesity (yes/no) initially and blood $\mathrm{Mg}$ (below $0.65 \mathrm{mmol} / 1,0.65-0.95 \mathrm{mmol} / \mathrm{l}$ and over $0.95 \mathrm{mmol} / \mathrm{l})$ afterward according to the classification of CHNS.

Additional information collected from interviews, physical examination and clinical records included the following: age, sex, weight, height, BMI, dyslipidaemia, dietary factors and use of anti-diabetic drugs (Fig. 1).

\section{Statistical methods}

Differences between $\mathrm{Mg}$ groups in socio-demographic and clinical characteristics were evaluated by ANOVA for continuous variables and $\chi^{2}$ test for categorical variables. In particular, we calculated and tested the differences in blood lipids among the three Mg groups and among T2D (yes/no) and central obesity (yes/no) subgroups.

A generalised linear model of blood lipids (TAG, TC, HDLcholesterol LDL-cholesterol) and Mg (three groups) was built 

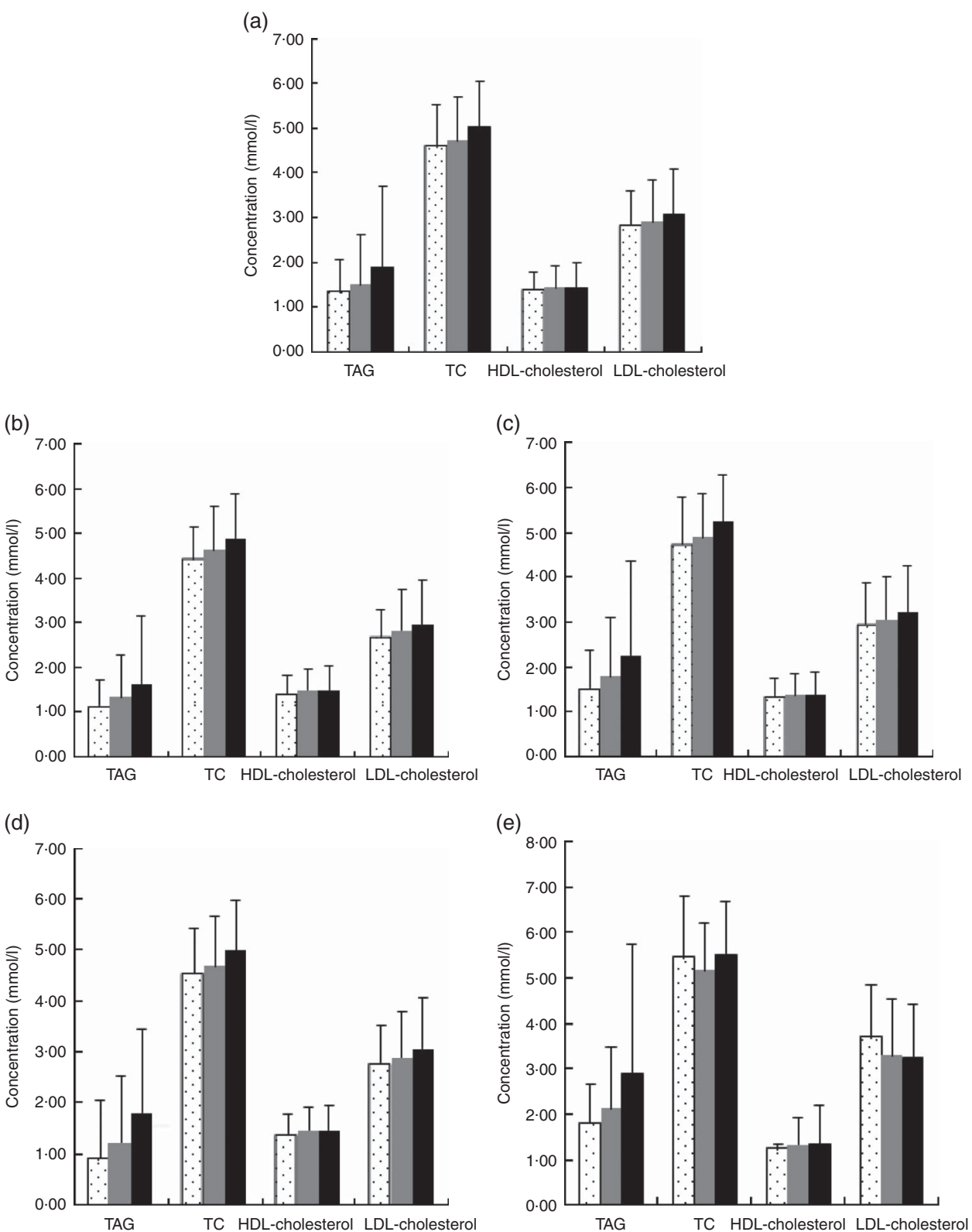

Fig. 1. Blood lipids according to type 2 diabetes (T2D) and central obesity status. (a) All subjects group, mean TAG, total cholesterol (TC), HDL-cholesterol, LDLcholesterol (1.68, 4.87, 1.44, $2.98 \mathrm{mmol} / \mathrm{l})$, (b) no central obesity group, mean TAG, TC, HDL-cholesterol, LDL-cholesterol $(1.47,4.74,1.48,2 \cdot 88 \mathrm{mmol} / \mathrm{l})$, (c) central obesity group, mean TAG, TC, HDL-cholesterol, LDL-cholesterol (2.02, 5.07, 1.37, 3.14 mmol/), (d) No T2D group, mean TAG, TC, HDL-cholesterol, LDL-cholesterol $(1.60,4.82,1.45,2.96 \mathrm{mmol} / \mathrm{l})$ and (e) T2D group, mean TAG, TC, HDL-cholesterol, LDL-cholesterol $(2.52,5.34,1.33,3.27 \mathrm{mmol} / \mathrm{l})$. Values are means and $95 \% \mathrm{Cl}$ represented by vertical bars. $\Theta$, Low magnesium $\leq 0.65 \mathrm{mmol} / / ; \square$, normal magnesium $0.65-0.95 \mathrm{mmol} / /$; $\square$, high magnesium $\geq 0.95$.

adjusting for covariates, which included age (continuous), sex, BMI (continuous), WC (continuous), T2D (yes/no), antidiabetic drug treatment (yes/no), insulin injection (yes/no), blood pressure(continuous), energy intake (continuous), fat intake (continuous), protein intake (continuous), carbohydrate intake (continuous), HbA1c (\%), urea (continuous), uric acid (continuous), apoA-1 (continuous), apoB (continuous), lipoprotein (continuous), creatinine (continuous) and insulin (continuous).

Separate adjusted models were also built for subjects with or without T2D. As a sensitivity analysis, we performed more parsimonious models, excluding adjustments for some variables dealing with possible collinearity among some covariates. $P$ values $<0.05$ were considered to be statistically significant.

\section{Results}

A total of 8163 subjects (mean age 50.9 years, $46.7 \%$ males) were included in this analysis. Most of them (91.7\%) were without T2D and $38.4 \%$ of the subjects had central obesity. The mean values of blood lipids (TAG, TC, HDL-cholesterol and LDL-cholesterol) were $1.33,4.59,1.38$ and $2.82 \mathrm{mmol} / \mathrm{l}$.

Approximately $8.3 \%$ of the population had T2D. As presented in Table 1, the serum Mg of $52 \cdot 2 \%$ was at a normal 
level, $1 \%$ at a low level $(\leq 0.65 \mathrm{mmol} / \mathrm{l})$ and $46.8 \%$ at a high level $(\geq 0.95 \mathrm{mmol} / \mathrm{l})$. Subjects with a high level of serum $\mathrm{Mg}$ were characterised by significantly older age $(P<0.0001)$, higher blood pressure $(P<0.0001)$ and a higher WC (in males) $(P<0 \cdot 0001)$, but these differences were not clinically meaningful.

As the level of serum Mg increased, there was a significantly higher prevalence of T2D and a higher biochemical indicator $(P<0 \cdot 01)$, with the exception of apoA-1 and lipoprotein values.

Table 2 shows blood lipid values in the whole group, according to central obesity category and $\mathrm{Mg}$ status. A significant increase in blood lipids, with the exception of HDL-cholesterol, across progressive $\mathrm{Mg}$ groups (from low $\mathrm{Mg}$ level $\leq 0.65 \mathrm{mmol} / \mathrm{l}$, normal level $0.65-0.95 \mathrm{mmol} / \mathrm{l}$ to high level $\geq 0.95 \mathrm{mmol} / \mathrm{l})$ in all subjects was noted $(P<0.05)$. This phenomenon was also detected in subgroups, regardless of whether they were with central obesity or not. In addition, when compared with those of the group without central obesity, the mean increments of TAG, TC, HDL-cholesterol and LDL-cholesterol of subjects with central obesity were 1.52, 4.75, 1.36 and $2.97 \mathrm{mmol} / \mathrm{l}$ in the group with $\mathrm{Mg}$ level $\leq 0.65 \mathrm{mmol} / \mathrm{l}$; $1.79,4.9,1.37$ and $3.07 \mathrm{mmol} / \mathrm{l}$ in the group with $\mathrm{Mg}$ level of $0.65-0.95 \mathrm{mmol} / \mathrm{l}$; and $2.26,5.25,1.36$ and $3.21 \mathrm{mmol} / \mathrm{l}$ in the group with $\mathrm{Mg}$ level $\geq 0.95 \mathrm{mmol} / \mathrm{l}$. TAG, TC, HDL-cholesterol and LDL-cholesterol were significantly higher among subjects with central obesity compared with those without central obesity $(P<0.05)$.

Data of subjects with and without T2D are presented in Table 3. As depicted in Table 3, a significant increase in TAG, TC and LDL-cholesterol across progressive Mg groups was observed in subjects without T2D $(P<0 \cdot 05)$. However, no significant change of HDL-cholesterol and LDL-cholesterol across

Table 1. Sample characteristics according to magnesium status

(Mean values and standard deviations; numbers and percentages)

\begin{tabular}{|c|c|c|c|c|c|c|c|c|c|}
\hline \multirow[b]{2}{*}{ Variables } & \multicolumn{2}{|c|}{ Total ( $n$ 8163) } & \multicolumn{2}{|c|}{$\begin{array}{c}\text { Low } \mathrm{Mg} \leq 0.65 \mathrm{mmol} / \mathrm{l} \\
(n 84)\end{array}$} & \multicolumn{2}{|c|}{$\begin{array}{c}\text { Normal Mg } \\
0.65-0.95 \mathrm{mmol} / \mathrm{l}(n \text { 4261) }\end{array}$} & \multicolumn{2}{|c|}{$\begin{array}{c}\text { High } \mathrm{Mg} \geq 0.95 \mathrm{mmol} / \mathrm{l} \\
(n 3818)\end{array}$} & \multirow[b]{2}{*}{$P$} \\
\hline & Mean & $\mathrm{SD}$ & Mean & SD & Mean & SD & Mean & SD & \\
\hline Age (years) & $50 \cdot 9$ & $15 \cdot 1$ & $48 \cdot 3$ & $14 \cdot 7$ & $49 \cdot 8$ & $15 \cdot 2$ & $52 \cdot 2$ & 14.9 & $<0.0001$ \\
\hline$\geq 60$ years & & & & & & & & & $<0.0001$ \\
\hline$n$ & \multicolumn{2}{|c|}{2275} & \multicolumn{2}{|c|}{18} & \multicolumn{2}{|c|}{1074} & \multicolumn{2}{|c|}{1183} & \\
\hline$\%$ & \multicolumn{2}{|c|}{27.9} & \multicolumn{2}{|c|}{$21 \cdot 4$} & \multicolumn{2}{|c|}{$25 \cdot 2$} & \multicolumn{2}{|c|}{$31 \cdot 0$} & \\
\hline \multicolumn{10}{|l|}{ Risk factors } \\
\hline Systolic pressure (mmHg) & $124 \cdot 9$ & $19 \cdot 0$ & $121 \cdot 0$ & $18 \cdot 5$ & $124 \cdot 1$ & $19 \cdot 2$ & $126 \cdot 0$ & $18 \cdot 7$ & $<0.0001$ \\
\hline Diastolic pressure $(\mathrm{mmHg})$ & $80 \cdot 3$ & $11 \cdot 2$ & $76 \cdot 6$ & $13 \cdot 2$ & 79.5 & $11 \cdot 3$ & $81 \cdot 2$ & $11 \cdot 0$ & $<0.0001$ \\
\hline BMI $\left(\mathrm{kg} / \mathrm{m}^{2}\right)$ & $23 \cdot 4$ & 3.5 & $23 \cdot 3$ & $3 \cdot 4$ & $23 \cdot 2$ & 3.5 & 23.6 & 3.5 & $<0.0001$ \\
\hline Obesity $\left(\mathrm{BMI} \geq 30 \mathrm{~kg} / \mathrm{m}^{2}\right)$ & & & & & & & & & 0.2223 \\
\hline$n$ & \multicolumn{2}{|c|}{338} & \multicolumn{2}{|c|}{2} & \multicolumn{2}{|c|}{168} & \multicolumn{2}{|c|}{168} & \\
\hline$\%$ & \multicolumn{2}{|c|}{$4 \cdot 14$} & \multicolumn{2}{|c|}{2.38} & \multicolumn{2}{|c|}{3.94} & & & \\
\hline Waist circumference (cm) & & & & & & & & & \\
\hline Men & 84.4 & $10 \cdot 2$ & $82 \cdot 6$ & $8 \cdot 3$ & $83 \cdot 6$ & $10 \cdot 1$ & $85 \cdot 2$ & $10 \cdot 3$ & $<0.0001$ \\
\hline Women & $81 \cdot 3$ & $10 \cdot 3$ & $84 \cdot 6$ & 11.4 & 80.5 & $10 \cdot 3$ & $82 \cdot 2$ & $10 \cdot 2$ & $<0.0001$ \\
\hline Central obesity & & & & & & & & & $<0.0001$ \\
\hline$n$ & & & & & & & & & \\
\hline$\%$ & & & & & & & & & \\
\hline T2D & & & & & & & & & 0.0069 \\
\hline$n$ & & & & & & & & & \\
\hline$\%$ & & & & & & & & & \\
\hline Dietary factors & & & & & & & & & \\
\hline Energy intake (kJ/d) & 8957.9 & $2753 \cdot 1$ & $9196 \cdot 4$ & $2456 \cdot 0$ & $8874 \cdot 3$ & 2774.0 & $9016 \cdot 5$ & $2736 \cdot 3$ & $0 \cdot 1618$ \\
\hline Energy intake (kcal/d) & $2141 \cdot 3$ & 658.7 & $2198 \cdot 8$ & 587.7 & $2121 \cdot 7$ & 663.6 & $2155 \cdot 2$ & 654.5 & $0 \cdot 1618$ \\
\hline Fat intake $(\mathrm{g} / \mathrm{d})$ & 75.4 & $39 \cdot 7$ & $82 \cdot 7$ & $31 \cdot 8$ & $75 \cdot 3$ & $40 \cdot 9$ & $75 \cdot 3$ & 38.5 & 0.2347 \\
\hline Protein intake $(\mathrm{g} / \mathrm{d})$ & $65 \cdot 8$ & 22.9 & $65 \cdot 4$ & $26 \cdot 4$ & $64 \cdot 7$ & $22 \cdot 5$ & $67 \cdot 0$ & $23 \cdot 2$ & $<0.0001$ \\
\hline Carbohydrate intake (g/d) & $295 \cdot 1$ & 101.4 & 291.3 & $108 \cdot 5$ & 293.2 & $102 \cdot 0$ & $297 \cdot 3$ & $100 \cdot 6$ & 0.2376 \\
\hline Analytical values & & & & & & & & & \\
\hline HbA1c (\%) & $5 \cdot 6$ & 0.9 & $5 \cdot 3$ & 0.5 & $5 \cdot 6$ & 1.0 & $5 \cdot 7$ & 0.8 & $<0.0001$ \\
\hline Urea $(\mathrm{mmol} / \mathrm{l})$ & 5.5 & 1.6 & $5 \cdot 3$ & 1.5 & $5 \cdot 3$ & 1.5 & $5 \cdot 6$ & $1 \cdot 7$ & $<0.0001$ \\
\hline Uric acid (mg/ml) & 3.083 & 1.063 & $2 \cdot 682$ & 0.831 & $2 \cdot 941$ & 0.900 & 3.252 & $1 \cdot 201$ & $<0.0001$ \\
\hline apoA-1 (g/l) & $1 \cdot 2$ & 0.4 & 1.0 & 0.2 & $1 \cdot 2$ & 0.4 & $1 \cdot 1$ & 0.4 & $<0.0001$ \\
\hline apoB $(g / l)$ & 0.9 & 0.3 & 0.8 & $0 \cdot 2$ & 0.9 & 0.3 & 0.9 & 0.3 & $<0.0001$ \\
\hline Lipoprotein (mg/ml) & 0.156 & 0.229 & 0.131 & 0.128 & 0.158 & 0.227 & 0.154 & 0.232 & 0.4193 \\
\hline Creatinine $(\mu \mathrm{mol} / \mathrm{l})$ & $87 \cdot 4$ & $22 \cdot 5$ & $85 \cdot 2$ & $13 \cdot 2$ & 85.4 & $17 \cdot 8$ & 89.7 & $26 \cdot 7$ & $<0.0001$ \\
\hline Insulin ( $\mu \mathrm{lU} / \mathrm{ml})$ & $14 \cdot 3$ & $22 \cdot 1$ & 8.4 & 8.6 & $13 \cdot 7$ & $20 \cdot 1$ & $15 \cdot 2$ & $24 \cdot 3$ & 0.0003 \\
\hline Anti-diabetic drugs & & & & & & & & & \\
\hline Oral drugs & & & & & & & & & 0.4467 \\
\hline$n$ & & & & & & & & & \\
\hline$\%$ & & & & & & & & & \\
\hline Insulin injection & & & & & & & & & 0.3672 \\
\hline$n$ & & & & & & & & & \\
\hline$\%$ & & & & & & & & & \\
\hline
\end{tabular}

T2D, type 2 diabetes. 
Table 2. Blood lipid values according to central obesity and magnesium status (Mean values and standard deviations)

\begin{tabular}{|c|c|c|c|c|c|c|c|c|c|c|c|c|c|c|c|c|c|c|c|c|c|}
\hline & \multicolumn{7}{|c|}{ Total } & \multicolumn{7}{|c|}{ Without central obesity } & \multicolumn{7}{|c|}{ With central obesity } \\
\hline & \multicolumn{2}{|c|}{$\begin{array}{c}\text { Low Mg } \\
\leq 0.65 \mathrm{mmol} / \mathrm{l} \\
\end{array}$} & \multicolumn{2}{|c|}{$\begin{array}{c}\text { Normal Mg } \\
0.65- \\
0.95 \mathrm{mmol} / \mathrm{l} \\
\end{array}$} & \multicolumn{2}{|c|}{$\begin{array}{c}\text { High Mg } \\
\geq 0.95 \mathrm{mmol} / \mathrm{l} \\
\end{array}$} & \multirow[b]{2}{*}{$P$} & \multicolumn{2}{|c|}{$\begin{array}{c}\text { Low Mg } \\
\leq 0.65 \mathrm{mmol} / \mathrm{l}\end{array}$} & \multicolumn{2}{|c|}{$\begin{array}{c}\text { Normal Mg } \\
0.65- \\
0.95 \mathrm{mmol} / \mathrm{l}\end{array}$} & \multicolumn{2}{|c|}{$\begin{array}{c}\text { High Mg } \\
\geq 0.95 \mathrm{mmol} / \mathrm{l} \\
\end{array}$} & \multirow[b]{2}{*}{$P$} & \multicolumn{2}{|c|}{$\begin{array}{c}\text { Low Mg } \\
\leq 0.65 \mathrm{mmol} / \mathrm{l}\end{array}$} & \multicolumn{2}{|c|}{$\begin{array}{l}\text { Normal Mg } \\
0.65- \\
0.95 \mathrm{mmol} / \mathrm{l}\end{array}$} & \multicolumn{2}{|c|}{$\begin{array}{c}\text { High Mg } \\
\geq 0.95 \mathrm{mmol} / /\end{array}$} & \multirow[b]{2}{*}{$P$} \\
\hline & Mean & SD & Mean & SD & Mean & SD & & Mean & SD & Mean & SD & Mean & SD & & Mean & $\mathrm{SD}$ & Mean & SD & Mean & SD & \\
\hline TAG ( & 1.33 & 0.74 & 1.50 & $1 \cdot 11$ & 1.89 & 1.81 & 0.0001 & $1 \cdot 14$ & 0.56 & 1.33 & 0.95 & 1.63 & 1.53 & 0.0001 & 1.52 & 0.86 & 1.79 & 1.30 & $2 \cdot 26$ & $2 \cdot 10$ & $<0.0001$ \\
\hline $\mathrm{TC}(\mathrm{mmol} / \mathrm{l})$ & 4.59 & 0.92 & 4.72 & 0.98 & 5.03 & 1.02 & $<0.0001$ & 4.42 & 0.72 & 4.63 & 0.96 & 4.89 & 0.98 & $<0.0001$ & 4.75 & 1.06 & 4.90 & 0.97 & $5 \cdot 25$ & 1.03 & $<0.0001$ \\
\hline HDL-cholesterol & 1.38 & 0.38 & 1.44 & 0.46 & 1.43 & 0.54 & 0.3340 & 1.40 & 0.40 & 1.49 & 0.45 & 1.48 & 0.54 & 0.5023 & 1.36 & 0.37 & 1.37 & 0.47 & 1.36 & 0.53 & 0.9265 \\
\hline LDL-cholesterol (mmol//) & $2 \cdot 82$ & 0.78 & 2.91 & 0.94 & 3.07 & 1.03 & $<0.0001$ & $2 \cdot 67$ & 0.62 & $2 \cdot 82$ & 0.92 & 2.96 & 1.01 & $<0.0001$ & 2.97 & 0.90 & 3.07 & 0.95 & 3.21 & 1.05 & 0.0004 \\
\hline
\end{tabular}

TC, total cholesterol.

Table 3. Blood lipid values according to central obesity and magnesium status in patients without type 2 diabetes (T2D) and in patients with T2D (Mean values and standard deviations)

\begin{tabular}{|c|c|c|c|c|c|c|c|c|c|c|c|c|c|c|c|c|c|c|c|c|c|}
\hline & \multicolumn{7}{|c|}{ Total } & \multicolumn{7}{|c|}{ Without central obesity } & \multicolumn{7}{|c|}{ With central obesity } \\
\hline & \multicolumn{2}{|c|}{$\begin{array}{c}\text { Low Mg } \\
\leq 0.65 \mathrm{mmol} / \mathrm{l}\end{array}$} & \multicolumn{2}{|c|}{$\begin{array}{c}\text { Normal Mg } \\
0.65- \\
0.95 \mathrm{mmol} / \mathrm{l}\end{array}$} & \multicolumn{2}{|c|}{$\begin{array}{c}\text { High Mg } \\
\geq 0.95 \mathrm{mmol} / \mathrm{l}\end{array}$} & \multirow[b]{2}{*}{$P$} & \multicolumn{2}{|c|}{$\begin{array}{c}\text { Low Mg } \\
\leq 0.65 \mathrm{mmol} / \mathrm{l}\end{array}$} & \multicolumn{2}{|c|}{$\begin{array}{c}\text { Normal Mg } \\
0.65- \\
0.95 \mathrm{mmol} / /\end{array}$} & \multicolumn{2}{|c|}{$\begin{array}{c}\text { High Mg } \\
\geq 0.95 \mathrm{mmol} / \mathrm{l}\end{array}$} & \multirow[b]{2}{*}{$P$} & \multicolumn{2}{|c|}{$\begin{array}{c}\text { Low Mg } \\
\leq 0.65 \mathrm{mmol} / \mathrm{l}\end{array}$} & \multicolumn{2}{|c|}{$\begin{array}{c}\text { Normal Mg } \\
0.65- \\
0.95 \mathrm{mmol} / \mathrm{l}\end{array}$} & \multicolumn{2}{|c|}{$\begin{array}{c}\text { High Mg } \\
\geq 0.95 \mathrm{mmol} / \mathrm{l}\end{array}$} & \\
\hline & Mean & SD & Mean & $\mathrm{SD}$ & Mean & SD & & Mean & SD & Mean & SD & Mean & SD & & Mean & SD & Mean & SD & Mean & SD & \\
\hline \multicolumn{22}{|l|}{ Subjects without T2D } \\
\hline $\mathrm{TAG}(\mathrm{mmol} / \mathrm{l})$ & 1.31 & 0.73 & 1.44 & 1.07 & 1.79 & 1.64 & $<0.0001$ & 1.12 & 0.53 & 1.31 & 0.94 & 1.57 & 1.37 & $<0.0001$ & 1.49 & 0.86 & 1.71 & 1.25 & $2 \cdot 14$ & 1.94 & $<0.0001$ \\
\hline $\mathrm{TC}(\mathrm{mmol} / \mathrm{l})$ & 4.54 & 0.88 & 4.69 & 0.96 & 4.99 & 0.99 & $<0.0001$ & 4.41 & 0.73 & 4.60 & 0.95 & 4.85 & 0.95 & $<0.0001$ & 4.68 & 1.00 & 4.86 & 0.96 & $5 \cdot 21$ & 1.02 & $<0.0001$ \\
\hline HDL-cholesterol $(\mathrm{mmol} / \mathrm{l})$ & 1.38 & 0.39 & 1.45 & 0.45 & 1.44 & 0.50 & 0.2558 & 1.40 & 0.41 & 1.49 & 0.42 & 1.49 & 0.55 & 0.5039 & 1.36 & 0.38 & 1.39 & 0.48 & 1.37 & 0.40 & 0.4363 \\
\hline LDL-cholesterol (mmol/l) & $2 \cdot 78$ & 0.74 & 2.88 & 0.90 & 3.05 & 1.02 & $<0.0001$ & $2 \cdot 67$ & 0.63 & $2 \cdot 79$ & 0.90 & 2.95 & 1.00 & $<0.0001$ & $2 \cdot 89$ & 0.83 & 3.05 & 0.88 & $3 \cdot 21$ & 1.03 & $<0.0001$ \\
\hline \multicolumn{22}{|l|}{ Subjects with T2D } \\
\hline $\mathrm{TAG}(\mathrm{mmol} / \mathrm{l})$ & 1.79 & 0.87 & $2 \cdot 13$ & 1.37 & 2.89 & 2.86 & $<0.0001$ & 1.62 & 1.04 & 1.78 & 1.07 & 2.73 & 3.05 & 0.0053 & 1.97 & 1.04 & 2.33 & 1.48 & 2.98 & $2 \cdot 76$ & 0.0110 \\
\hline $\mathrm{TC}(\mathrm{mmol} / \mathrm{l})$ & 5.44 & 1.37 & $5 \cdot 16$ & 1.07 & $5 \cdot 50$ & $1 \cdot 16$ & 0.0004 & 4.60 & 0.76 & $5 \cdot 18$ & $1 \cdot 15$ & 5.54 & 1.25 & 0.0418 & $6 \cdot 29$ & 1.47 & $5 \cdot 15$ & 1.02 & 5.48 & $1 \cdot 12$ & 0.0030 \\
\hline HDL-cholester & 1.23 & 0.09 & 1.32 & 0.59 & 1.35 & 0.85 & 0.8596 & 1.30 & 0.01 & 1.44 & 0.85 & 1.35 & 0.40 & 0.5834 & 1.17 & 0.06 & 1.25 & 0.35 & 1.34 & 1.02 & 0.4458 \\
\hline LDL-cholesterol (mmol/l) & 3.68 & $1 \cdot 17$ & 3.30 & 1.23 & 3.23 & 1.19 & 0.5658 & 2.80 & 0.36 & 3.37 & $1 \cdot 11$ & 3.28 & 1.24 & 0.6938 & 4.56 & 0.92 & 3.26 & 1.30 & $3 \cdot 20$ & $1 \cdot 16$ & 0.2620 \\
\hline
\end{tabular}

TC, total cholesterol. 
progressive $\mathrm{Mg}$ groups was observed in subjects with T2D $(P>0.05)$. In addition, TAG, TC, HDL-cholesterol and LDLcholesterol were significantly higher among subjects with T2D than those without T2D $(P<0 \cdot 05)$.

\section{Multivariable analysis of blood lipids with magnesium}

The generalised linear model showed that after full adjustment of demographic characteristics, lifestyles, dietary factors and clinic variables (including T2D, blood glucose, level, antidiabetic drugs treatment, insulin injection, blood pressure, Urea, Uric Acid, apoA-1, apoB, lipoprotein, creatinine, insulin), TAG, TC, HDL-cholesterol and LDL-cholesterol were significantly higher among subjects with a higher level of $\mathrm{Mg} \geq 0.95 \mathrm{mmol} / \mathrm{l}$ than those with a lower level $(P<0.05)$. According to the $95 \%$ $\mathrm{CI}$, there was no significant difference between subjects with a serum $\mathrm{Mg}$ level $\leq 0.65 \mathrm{mmol} / \mathrm{l}$ and those with a level of 0.65-0.95 mmol/1.

Multivariable models for TAG and LDL-cholesterol failed to attain statistical significance in subjects with T2D. When the parsimonious model was used, the aforementioned general pattern was quite similar (Table 4). Therefore, this phenomenon was noted to be quite similar among subjects with T2D, by using a generalised linear model or parsimonious model.

\section{Discussion}

There were significant correlations between abdominal obesity indicators and lipid levels in previous studies ${ }^{(21,22)}$. Our study supported this view that there was a positive correlation between serum lipids and central obesity $(P<0 \cdot 05)$. Thus, the central obesity factor needs to be adjusted to, while detecting the relationship between $\mathrm{Mg}$ and lipids.

The previous study shows that low Mg levels are related to T2D mellitus and its complications ${ }^{\text {(23) }}$. It is believed that low $\mathrm{Mg}$ levels are conducive to the development of insulin resistance ${ }^{(24,25)}$. The role of low Mg levels in the development of diabetic complications has been convincingly presented by Corsonello et $a l^{(26)}$ and $\mathrm{Xu}$ et $a l^{(27)}$, who suggested that serum concentrations of $\mathrm{Mg}$ correlate inversely with microalbuminuria. In the study by Rasheed et $a l^{(17)}$, serum $\mathrm{Mg}$ in diabetic patients was correlated with all lipid parameters.

Table 4. Generalised linear model of the association between blood lipids and magnesium according to type 2 diabetes (T2D) status

(Mean values and $95 \%$ confidence intervals)

\begin{tabular}{|c|c|c|c|c|c|c|c|}
\hline & \multicolumn{6}{|c|}{ Mg groups } & \multirow[b]{3}{*}{$P$} \\
\hline & \multicolumn{2}{|c|}{$\leq 0.65 \mathrm{mmol} / \mathrm{l}$} & \multicolumn{2}{|c|}{$0.65-0.95 \mathrm{mmol} / \mathrm{l}$} & \multicolumn{2}{|c|}{$\geq 0.95 \mathrm{mmol} / \mathrm{l}$} & \\
\hline & Mean & $95 \% \mathrm{Cl}$ & Mean & $95 \% \mathrm{Cl}$ & Mean & $95 \% \mathrm{Cl}$ & \\
\hline \multicolumn{8}{|l|}{ Full model* } \\
\hline \multicolumn{8}{|l|}{ Total } \\
\hline TAG (mmol/l) & 1.75 & $1.51,1.98$ & 1.60 & $1.54,1.66$ & 1.82 & $1.76,1.88$ & $<0.0001$ \\
\hline $\mathrm{TC}(\mathrm{mmol} / \mathrm{l})$ & 4.92 & $4.80,5.04$ & $4 \cdot 80$ & $4.80,4.83$ & 4.97 & $4.94,5.00$ & $<0.0001$ \\
\hline HDL-cholesterol (mmol/l) & 1.40 & $1.31,1.48$ & 1.40 & $1.38,1.42$ & 1.45 & $1.43,1.48$ & $<0.0001$ \\
\hline LDL-cholesterol (mmol/l) & 3.00 & $2.87,3.13$ & 2.93 & $2.90,2.97$ & 2.98 & $2.94,3.01$ & 0.0068 \\
\hline \multicolumn{8}{|l|}{ Subjects without T2D } \\
\hline TAG (mmol/l) & 1.61 & $1.36,1.87$ & 1.50 & $1.46,1.54$ & 1.67 & $1.62,1.71$ & $<0.0001$ \\
\hline $\mathrm{TC}(\mathrm{mmol} / \mathrm{l})$ & 4.89 & $4.77,5.01$ & 4.77 & $4.75,4.79$ & 4.93 & $4.91,4.95$ & $<0.0001$ \\
\hline HDL-cholesterol $(\mathrm{mmol} / \mathrm{l})$ & 1.43 & $1.34,1.52$ & 1.43 & $1.42,1.45$ & 1.48 & $1.46,1.49$ & $<0.0001$ \\
\hline LDL-cholesterol (mmol/l) & 3.01 & $2.89,3.14$ & 2.96 & $2.94,2.98$ & 3.01 & $2.99,3.03$ & 0.0029 \\
\hline \multicolumn{8}{|l|}{ Subjects with T2D } \\
\hline TAG (mmol/l) & 1.38 & $-0.39,3.14$ & 2.33 & $2 \cdot 12,2.54$ & 2.58 & $2 \cdot 37,2.79$ & 0.0845 \\
\hline $\mathrm{TC}(\mathrm{mmol} / \mathrm{l})$ & 5.38 & $4.68,6.08$ & 5.22 & $5 \cdot 14,5 \cdot 31$ & 5.45 & $5 \cdot 37,5.53$ & 0.0002 \\
\hline HDL-cholesterol $(\mathrm{mmol} / \mathrm{l})$ & 1.30 & $0.98,1.62$ & 1.26 & $1.23,1.30$ & 1.36 & $1.32,1.40$ & 0.0006 \\
\hline LDL-cholesterol (mmol/l) & 3.85 & $2.92,4.77$ & 3.33 & $3.22,3.44$ & 3.26 & $3.15,3.37$ & 0.3087 \\
\hline \multicolumn{8}{|l|}{ Parsimonious model } \\
\hline \multicolumn{8}{|l|}{ Total } \\
\hline TAG (mmol/l) & 1.78 & $1.51,2.04$ & 1.68 & $1.61,1.74$ & 1.85 & $1.78,1.92$ & $<0.0001$ \\
\hline $\mathrm{TC}(\mathrm{mmol} / \mathrm{l})$ & 4.91 & $4.80,5.03$ & 4.80 & $4.78,4.82$ & 4.97 & $4.96,4.99$ & $<0.0001$ \\
\hline HDL-cholesterol $(\mathrm{mmol} / \mathrm{l})$ & 1.39 & $1.30,1.50$ & 1.40 & $1.38,1.42$ & 1.46 & $1.44,1.48$ & $<0.0001$ \\
\hline LDL-cholesterol $(\mathrm{mmol} / \mathrm{l})$ & 2.99 & $2 \cdot 86,3 \cdot 12$ & 2.93 & $2.90,2.96$ & 2.97 & $2.94,3.00$ & 0.0076 \\
\hline \multicolumn{8}{|l|}{ Subjects without T2D } \\
\hline TAG (mmol/l) & 1.60 & $1.35,1.85$ & 1.50 & $1.46,1.54$ & 1.66 & $1.62,1.70$ & $<0.0001$ \\
\hline $\mathrm{TC}(\mathrm{mmol} / \mathrm{l})$ & $4 \cdot 88$ & $4 \cdot 76,5 \cdot 00$ & 4.77 & $4.75,4.79$ & 4.93 & $4.91,4.95$ & $<0.0001$ \\
\hline HDL-cholesterol $(\mathrm{mmol} / \mathrm{l})$ & 1.42 & $1.33,1.51$ & 1.42 & $1.41,1.44$ & 1.47 & $1.46,1.49$ & $<0.0001$ \\
\hline LDL-cholesterol $(\mathrm{mmol} / \mathrm{l})$ & 3.00 & $2 \cdot 88,3 \cdot 12$ & 2.95 & $2.94,2.97$ & 3.00 & $2.98,3.02$ & 0.0021 \\
\hline \multicolumn{8}{|l|}{ Subjects with T2D } \\
\hline TAG (mmol/l) & 1.38 & $-0.37,3.13$ & $2 \cdot 33$ & $2 \cdot 15,2.51$ & 2.62 & $2 \cdot 45,2.79$ & 0.0332 \\
\hline $\mathrm{TC}(\mathrm{mmol} / \mathrm{l})$ & $5 \cdot 43$ & $4 \cdot 73,6 \cdot 13$ & $5 \cdot 26$ & $5 \cdot 19,5 \cdot 33$ & $5 \cdot 45$ & $5 \cdot 38,5 \cdot 52$ & 0.0013 \\
\hline HDL-cholesterol $(\mathrm{mmol} / \mathrm{l})$ & 1.27 & $0.63,1.91$ & 1.25 & $1.18,1.32$ & 1.39 & $1.32,1.46$ & 0.0290 \\
\hline LDL-cholesterol (mmol/l) & 3.62 & $2.88,4.37$ & 3.29 & $3.21,3.37$ & $3 \cdot 24$ & $3 \cdot 16,3 \cdot 32$ & 0.4404 \\
\hline
\end{tabular}

TC, total cholesterol.

* Models were adjusted for demographic characteristics, lifestyles, dietary factors and clinic variables (including T2D, blood glucose, level, anti-diabetic drugs treatment, insulin injection, blood pressure, urea, uric acid, apoA-1, apoB, lipoprotein, creatinine and insulin).

†The parsimonious model adjusted for the same variables as the full model except for some variables dealing with possible collinearity among some covariates. 
Among them, HDL-cholesterol was significantly $(P<0.05)$ positively correlated $(r 0.34)$, whereas TC and LDL-cholesterol were negatively correlated, albeit non-significantly, with serum $\mathrm{Mg}$.

However, Jiancheng et ll $^{(28)}$ failed to show any association between serum $\mathrm{Mg}$ and lipid profile in diabetic patients with or without complications. Meanwhile, Guerrero-Romero \& Rodriguez-Moran ${ }^{(29)}$ showed that in patients with T2D mellitus hypomagnesaemia is linked with low levels of HDL, irrespective of serum glucose level.

In our study, a significant increase of HbA1c (\%), insulin and blood lipids, with the exception of HDL-cholesterol, across progressive $\mathrm{Mg}$ groups in all subjects was noted $(P<0.05)$. T2D hindered the positive association between LDL-cholesterol and serum Mg. However, this positive association was still noted in subjects without T2D.

Lipid disturbance (higher TAG, TC and LDL with lower HDL) is most pronounced in diabetic children with hypomagnesaemia compared with those with normal serum $\mathrm{Mg}$. A negative correlation is noted between serum Mg and TAG $(r$ 0.636, $P<0.001)$, TC $(r 0.743, P<0.001)$ and LDL $(r 0.634, P<0.001)$. However, there was a positive correlation between serum $\mathrm{Mg}$ and HDL $(r 0.639, P<0.001)^{(30)}$. Our study results are consistent with this study on type 2 diabetic patients by using a generalised linear model or parsimonious model.

Meanwhile, statistically significant positive correlations between $\mathrm{Mg}$ concentration and TC or LDL concentration were found in middle-aged and older men ${ }^{(31)}$. These results are in concordance with the study by Mishra et $a l^{(32)}$ on type 2 adult diabetic patients that revealed a negative correlation between serum Mg and TAG $(r 0.519, P<0.01)$ and a positive correlation between serum Mg and HDL $(r$ 0.741), but did not show any significant correlation between TC or LDL with serum $\mathrm{Mg}$. Moreover, Srinivasan et $a l .{ }^{(24)}$ revealed a negative correlation between serum $\mathrm{Mg}$ and TAG $(P<0.05)$, especially in poorly controlled diabetics $\left(r\right.$ 0.632), and Rasheed et al. ${ }^{(33)}$ revealed a positive correlation between serum $\mathrm{Mg}$ and HDL $(r$ 0.34, $P<0 \cdot 01)$, but there was a non-significant correlation with other lipid parameters.

Guerrero-Romero \& Rodriguez-Moran ${ }^{(34)}$ suggests that hypomagnesaemia contributes to lower HDL. Similarly, Randell et $a{ }^{(35)}$ found positive correlations between $\mathrm{Mg}$ and $\mathrm{TC}$ and between $\mathrm{Mg}$ and LDL. In contrast, the results of Mahalle et al. ${ }^{(36)}$ indicate that lower Mg levels are associated with higher TC and LDL, and lower HDL.

Our study results are quite similar to Mahalle's view, by using generalised linear model or parsimonious model. Some of the conflicting studies whose results (lower Mg levels associated with lower blood lipids) confused us might be attributed to insufficient adjustment for confounders. In our study, there was no significant difference between subjects with the serum $\mathrm{Mg}$ level $\leq 0.65 \mathrm{mmol} / 1$ and those with the level of $0.65-0.95 \mathrm{mmol} / \mathrm{l}$, by using a generalised linear model or parsimonious model.

Many studies show the relationship between $\mathrm{Mg}$ intake or status, and the prevalence of chronic diseases: hypertension, lipid disorders and the presence of CVD and the metabolic syndrome ${ }^{(37,38)}$. Nevertheless, in a study on patients with the metabolic syndrome, $\mathrm{Mg}$ supplementation (3 months, $400 \mathrm{mg} / \mathrm{d}$ ) had no effect on improving lipid and carbohydrate metabolism ${ }^{(39)}$.
However, in patients with T2D, 3 months of oral Mg supplementation resulted in significantly reduced LDL and fasting glucose compared with the control group receiving placebo ${ }^{(40)}$. Although there was no $\mathrm{Mg}$ supplement in our study, one thing is for sure, T2D hindered the metabolism between $\mathrm{Mg}$ and blood lipids according to our study and these papers. This effect can be explained by the role of $\mathrm{Mg}$ in the activity of lipoprotein lipase enzyme as described by Rayssiguire and colleagues ${ }^{(41)}$. Meanwhile, there is a close correlation between T2D and lipase enzyme activity ${ }^{(42)}$.

Our research has several limitations. First, although we have corrected a number of confounding factors, we lack data regarding blood $n-3$ levels in our study, which may affect the blood lipid profile. Second, further studies are necessary to explore the precise reasons for the observed metabolism change influenced by T2D. Third, we lack data regarding lipoprotein lipase enzyme levels in our study, which may explore fundamental metabolism. Fourth, we lack data regarding Mg intake from diet. Fifth, the cross-sectional analysis of data does not allow causal assessment of the relationship studied. Sixth, despite exhaustive adjustments and stratifications used, we recognise that animal experiments are necessary to prove the results. We hope that future studies are conducted to address this area.

In summary, because a positive correlation between serum lipids and $\mathrm{Mg}$ was observed, $\mathrm{Mg}$ status in high level $(\geq 0.95 \mathrm{mmol} / \mathrm{l})$ is an adverse signal for high blood lipids. $\mathrm{Mg}$ status at a high level $(\geq 0.95 \mathrm{mmol} / \mathrm{l})$ is often accompanied by the high blood lipids, as shown in Table 4. In Table 4, the results were adjusted for most of the confounders in this research. As T2D hindered the metabolism between $\mathrm{Mg}$ and blood lipids through lipase enzyme activity, prevention of T2D is very important in the protection of endocrine function. TAG, TC, HDL-cholesterol and LDL-cholesterol were significantly higher among subjects with T2D or central obesity than those without T2D or central obesity $(P<0 \cdot 05)$. Meanwhile, the level of blood lipids (with the exception of HDL-cholesterol) was associated with that of serum $\mathrm{Mg}$, but this association was somehow hindered by T2D in LDL-cholesterol. In addition, both multivariable models for TAG and LDL-cholesterol failed to attain statistical significance among subjects with T2D, different from those without T2D. This new finding is attributed to our sufficient adjustment for confounders.

\section{Acknowledgements}

This research uses data from the China Health and Nutrition Survey (CHNS). The authors thank the National Institute for Nutrition and Health, China Center for Disease Control and Prevention, Carolina Population Center (P2C HD050924, T32 HD007168), the University of North Carolina at Chapel Hill, the National Institutes of Health (NIH) (R01-HD30880, DK056350, R24 HD050924 and R01-HD38700) and the NIH Fogarty International Center (D43 TW009077, D43 TW007709) for financial support for the CHNS data collection and analysis files from 1989 to 2015 and future surveys, and the China-Japan Friendship Hospital, Ministry of Health for support for CHNS 2009, Chinese National Human Genome Center at Shanghai since 2009 and Beijing Municipal Center for Disease Prevention and Control since 2011. 
L. Y., J. Z. and L. W. contributed to the study concept, design and supervision; the analysis and interpretation of the data; and the drafting and critical revision of the manuscript for important intellectual content. S. L., Q. Z. and P. X. contributed to the analysis and interpretation of the data, and to critical revision of the manuscript for important intellectual content. K. W., M. Z. and Y. J. contributed to administrative, technical and material support for the study; to the analysis and interpretation of the data; and to critical revision of the manuscript for important intellectual content.

The authors declare that there are no conflicts of interest.

\section{References}

1. de Baaij JH, Hoenderop JG \& Bindels RJ (2015) Magnesium in man: implications for health and disease. Physiol Rev 95, $1-46$.

2. Chakraborti S, Chakraborti T, Mandal M, et al. (2002) Protective role of magnesium in cardiovascular diseases: a review. Mol Cell Biochem 238, 163-179.

3. Mooren FC (2015) Magnesium and disturbances in carbohydrate metabolism. Diabetes Obes Metab 17, 813-823.

4. Gröber U, Schmidt J \& Kisters K (2015) Magnesium in prevention and therapy. Nutrients 7, 8199-8226.

5. Joosten MM, Gansevoort RT, Mukamal KJ, et al. (2013) Urinary and plasma magnesium and risk of ischemic heart disease. $\mathrm{Am}$ J Clin Nutr 97, 1299-1306.

6. Del Gobbo LC, Imamura F, Wu JH, et al. (2013) Circulating and dietary magnesium and risk of cardiovascular disease: a systematic review and metaanalysis of prospective studies. Am J Clin Nutr 98, 160-173.

7. Qu X, Jin F, Hao Y, et al. (2013) Magnesium and the risk of cardiovascular events: a meta-analysis of prospective cohort studies. PLOS ONE 8, e57720.

8. Bertinato J, Wang KC \& Hayward S (2017) Serum magnesium concentrations in the Canadian population and associations with diabetes, glycemic regulation, and insulin resistance. Nutrients 9, E296.

9. Wilson PW, Meigs JB, Sullivan L, et al. (2007) Prediction of incident diabetes mellitus in middle-aged adults: the Framingham Offspring Study. Arch Intern Med 167, 1068-1074.

10. Expert Panel on Detection, Evaluation, and Treatment of High Blood Cholesterol in Adults (2001) Executive Summary of the Third Report of the National Cholesterol Education Program (NCEP) Expert Panel on Detection, Evaluation, and Treatment of High Blood Cholesterol In Adults (Adult Treatment Panel III). JAMA 285, 2486-2497.

11. Ley SH, Harris SB, Mamakeesick M, et al. (2009) Metabolic syndrome and its components as predictors of incident type 2 diabetes mellitus in an Aboriginal community. CMAJ 180, 617-624.

12. Song Q, Liu X, Wang A, et al. (2016) Associations between non-traditional lipid measures and risk for type 2 diabetes mellitus in a Chinese community population: a crosssectional study. Lipids Health Dis 15, 70.

13. Njelekela MA, Negishi H, Nara Y, et al. (2002) Obesity and lipid profiles in middle aged men and women in Tanzania. East Afr Med J 79, 58-64.

14. Bijari B, Taheri F, Chahkandi T, et al. (2015) The relationship between serum lipids and obesity among elementary school in Birjand: a case control study. $J$ Res Health Sci 15, 83-87.

15. Cao Y, Wang C, Guan K, et al. (2016) Association of magnesium in serum and urine with carotid intima-media thickness and serum lipids in middle-aged and elderly Chinese: a community-based cross-sectional study. Eur J Nutr 55 , 219-226.

16. Shahbah D, El Naga AA, Hassan T, et al. (2016) Status of serum magnesium in Egyptian children with type 1 diabetes and its correlation to glycemic control and lipid profile. Medicine (Baltimore) 95, e5166.

17. Rasheed H, Elahi S \& Ajaz H (2012) Serum magnesium and atherogenic lipid fractions in type II diabetic patients of Lahore, Pakistan. Biol Trace Elem Res 148, 165-169.

18. Popkin BM, Du S, Zhai F, et al. (2010) Cohort profile: the China health and nutrition survey - monitoring and understanding socioeconomic and health change in China, 1989-2011. Int J Epidemiol 39, 1435-1440.

19. Than NN, Soe HH, Palaniappan SK, et al. (2017) Magnesium for treating sickle cell disease. Cochrane Database Syst Rev, issue 4, CD011358.

20. Russo I, Traversa M, Bonomo K, et al. (2010) In central obesity, weight loss restores platelet sensitivity to nitric oxide and prostacyclin. Obesity (Silver Spring) 18, 788-797.

21. Rocha FL, Menezes TN, Melo RL, et al. (2013) Correlation between indicators of abdominal obesity and serum lipids in the elderly. Rev Assoc Med Bras (1992) 59, 48-55 (in English, Portuguese).

22. Ali O, Cerjak D, Kent JW, et al. (2014) Obesity, central adiposity and cardiometabolic risk factors in children and adolescents: a family-based study. Pediatr Obes 9, e58-e62.

23. Tossiello L (1996) Hypomagnesemia and diabetes mellitus: a review of clinical implications. Arch Int Med 156, $1143-1148$

24. Srinivasan AR, Niranjan G, Kuzhandai Velu V, et al. (2012) Status of serum magnesium in type 2 diabetes mellitus with particular reference to serum triacylglycerol levels. Diabetes Metab Syndr 6, 187-189.

25. Choi WS, Kim SH \& Chung JH (2014) Relationships of hair mineral concentrations with insulin resistance in metabolic syndrome. Biol Trace Elem Res 158, 323-329.

26. Corsonello A, Ientile R, Buemi M, et al. (2000) Serum ionized magnesium levels in type 2 diabetic patients with microalbuminuria or clinical proteinuria. Am J Nephrol 20, 187-192.

27. Xu B, Sun J, Deng X, et al. (2013) Low serum magnesium level is associated with microalbuminuria in Chinese diabetic patients. Int J Endocrinol 2013, 580685.

28. $\mathrm{Xu} \mathrm{J}, \mathrm{Xu} \mathrm{W}, \mathrm{Yao} \mathrm{H}$, et al. (2013) Associations of serum and urinary magnesium with the pre-diabetes, diabetes and diabetic complications in the Chinese Northeast population. PLOS ONE 8, e56750.

29. Guerrero-Romero F \& Rodriguez-Moran M (2000) Hypomagnesemia is linked to low serum HDL-cholesterol irrespective of serum glucose values. $J$ Diabetes Complications 14, 272-276.

30. Shahbah D, El Naga AA, Hassan T, et al. (2016) Status of serum magnesium in Egyptian children with type 1 diabetes and its correlation to glycemic control and lipid profile. Medicine (Baltimore) 95, e5166.

31. Rotter I, Kosik-Bogacka D, Dołęgowska B, et al. (2015) Relationship between serum magnesium concentration and metabolic and hormonal disorders in middle-aged and older men. Magnes Res 28, 99-107.

32. Mishra S, Padmanaban P, Deepti GN, et al. (2012) Serum magnesium, dyslipidemia in type-2 diabetes mellitus. Biomed Res 23, 295-300.

33. Rasheed H, Elahi S \& Ajaz H (2012) Serum magnesium and atherogenic lipid fractions in type II diabetic 
patients of Lahore, Pakistan. Biol Trace Elem Res 148 165-169.

34. Guerrero-Romero F \& Rodriguez-Moran M (2002) Low serum magnesium levels and metabolic syndrome. Acta Diabetol 39 , 209-213.

35. Randell EW, Mathews M, Gadag V, et al. (2008) Relationship between serum magnesium values, lipids and anthropometric risk factors. Atherosclerosis 196, 413-419.

36. Mahalle N, Kulkarni MV \& Naik SS (2012) Is hypomagnesaemia a coronary risk factor among Indians with coronary artery disease? J Cardiovasc Dis Res 3, 280-286.

37. Swaminathan R (2003) Magnesium metabolism and its disorders. Clin Biochem Rev 24, 47-66.

38. Huang JH, Lu YF, Cheng FC, et al. (2012) Correlation of magnesium intake with metabolic parameters, depression and physical activity in elderly type 2 diabetes patients: a crosssectional study. Nutr J 13, 11-41.

39. Lima de Souza E, Silva Mde L, Cruz T, et al. (2014) Magnesium replacement does not improve insulin resistance in patients with metabolic syndrome: a 12-week randomized doubleblind study. J Clin Med Res 6, 456-462.

40. Solati M, Ouspid E, Hosseini S, et al. (2014) Oral magnesium supplementation in type II diabetic patients. Med J Islam Repub Iran 15, 28-67.

41 Rayssiguier Y \& Gueux E (1986) Magnesium and lipids in cardiovascular disease. $J$ Am Coll Nutr 5, 507-519.

42. Liu D, Gao H, Tang W, et al. (2017) Plant non-starch polysaccharides that inhibit key enzymes linked to type 2 diabetes mellitus. Ann N Y Acad Sci 1401, 28-36. 